\title{
Archaeal DNA polymerases: new frontiers in DNA replication and repair
}

Christopher D. O. Cooper ${ }^{1^{*}}$

${ }^{1}$ Department of Biological and Geographical Sciences, School of Applied Sciences, University of Huddersfield, Queensgate, Huddersfield, West Yorkshire, HD1 3DH, United Kingdom.

"Corresponding author:

c.d.cooper@hud.ac.uk

Tel: +44 (0)1484 471736

\section{Running title}

Archaeal DNA polymerases

\section{Keywords}

Archaea; DNA polymerase; exonuclease; DNA replication; DNA repair; replisome; holoenzyme; polymerase chain reaction; sequencing; biotechnology; polB1; Dpo1; PolB; PolD; PCNA, RFC.

\begin{abstract}
Archaeal DNA polymerases have long been studied due to their superior properties for DNA amplification in the Polymerase Chain Reaction and DNA sequencing technologies. However a full comprehension of their functions, recruitment and regulation as part of the replisome during genome replication and DNA repair lags behind well-established bacterial and eukaryotic model systems. The archaea are evolutionarily very broad, but a number of studies in the major model systems of both Crenarchaeota and Euryarchaeota are starting to yield significant increases in understanding of the functions of DNA polymerases in the respective phyla. Recent advances in biochemical approaches and in archaeal genetic models allowing knockout and epitope tagging have led to significant increases in our understanding, including DNA polymerase roles in Okazaki fragment maturation on the lagging strand, towards reconstitution of the replisome itself. Furthermore, poorly characterised DNA polymerase paralogues are finding roles in DNA repair and CRISPR immunity. This review attempts to provide a current update on the roles of archaeal DNA polymerases in both DNA replication and repair, addressing significant questions that remain for this field.
\end{abstract}




\section{Introduction}

All forms of life face a requirement to replicate their genomes in an accurate and timely manner [1]. Furthermore, DNA may be damaged following both endogenous and exogenous insults, with mutations potentially resulting if the lesions are not corrected by specific repair pathways, prior to DNA replication. Many archaea inhabit physical or chemical extremes, so could be expected to experience significant levels of exogenously-induced damage [2], therefore archaea are presumed to encode resilient DNA repair mechanisms [3]. Organisms hence require DNA polymerases, not only to synthesise daughter copies of genomic DNA during replication, but to also fill across gaps arising from lesion removal during DNA repair.

Since the discovery of Pol I from Escherichia coli in the 1950's [4], although much research has been undertaken into DNA polymerases and their roles in replication and repair, a significant force driving their study has been their biotechnological exploitation as reagents for DNA sequencing and DNA amplification in the Polymerase Chain Reaction (PCR). DNA polymerases from thermophilic archaea in particular have been well-studied (e.g. Pfu from Pyrococcus furiosus), generally being equipped with a 3'-5' exonuclease activity allowing them to 'proofread' DNA synthesis, ensuring accurate DNA amplification [5]. Significant advances have also been made in engineering archaeal polymerases for biotechnology, including fusion to DNA binding domains to enhance DNA synthesis processivity [6], and mutagenesis to allow incorporation of modified [7] or even artificial nucleotides [8]. Such mutagenesis approaches have been key to next-generation sequencing approaches [5,9], for instance, specific variants of Thermococcus sp. $9^{\circ} \mathrm{N}$ PolB DNA polymerase are able to incorporate bulky reversible dye-terminators required for sequencing-by-synthesis approaches, such as Illumina.

DNA polymerases are classified into 6 families ( $A, B, C, D, X$ and $Y$ ) and comprise a conserved nucleotidyltransferase catalytic mechanism, with strongly conserved motifs for both polymerase and exonuclease activities $[10,11]$. These DNA polymerase families share a core structure, shaped like a cupped right hand that encompasses the DNA primer-template $[12,13]$. This comprises several sub-domains important for catalysis (thumb, fingers, palm), with some appended specialised domains, such as the uracil binding domain in some Family $B$ polymerases [14]. Family B polymerases are found across all cellular life and viruses, and at least one Family B polymerase (PolB) is present in all archaea (Figure 1) [15,16]. Biochemical analysis and genome sequencing however soon indicated the presence of $Y$ family polymerases in some Crenarchaeota [17], and a non-canonical DNA polymerase (Family D, PolD) in the Euryarchaeota (Figure 1) [18]. Unusually, PolD is a heterodimer comprising a smaller DP1 3'-5' exonuclease subunit and the DP2 DNA polymerase subunit [18], and has also been successfully applied to PCR [19]. The evolutionary relationship of PolD to other DNA polymerases is unclear, although DP1 is thought to be ancestral to eukaryotic Family B polymerase structural subunits [15], but the DP2 sequence is very different to the canonical DNA polymerases. However, the recent structural determination of PolD [20] demonstrates that the DP2 subunit has a double-psi $\beta$-barrel fold conserved in multi-subunit RNA polymerases, potentially bridging DNA replication and transcription, rather than originating from a highly-divergent Family B polymerase $[15,20]$. 
Archaea also encode primases, DNA-dependent RNA polymerases recruited to origins to initiate DNA replication by de novo synthesis of RNA primers [21]. Archaeal primases are similar to eukaryotic primases, originally thought be comprise a heterodimer of catalytic PriS and regulatory PriL subunits (Figure 1) [22]. However recent studies suggest a third subunit PriX is important for primase activity, albeit phylogenetically restricted to some Crenarchaeota (Figure 1) [23]. Surprisingly, archaeal primases can catalyse not only RNA but also DNA synthesis, able to replicate up to several kilobases of product $[22,24]$ and so maybe classed as non-canonical DNA polymerases. Hence, primases are thought to sequentially hand-off between RNA and DNA synthesis [25], prior to transferring to a DNA polymerase. Putative bacterial-like DnaG primases are also found in archaeal genomes [26,27], although they appear to be associated with RNA degradation rather than replication $[27,28]$.

Archaeal DNA polymerase phylogenetic relationships are complex, as many Crenarchaeota contain two additional Family B members (polB2/Dpo2 and polB3/Dpo3) resulting from gene duplications, in addition to the polB1 (Dpo1) presumed major replicase (Figure 1) [29,30]. Furthermore, PolB from Euryarchaeota are more closely related to polB3 [15], suggesting polB1 and polB2 were derived from an ancestral polB3 following the Euryarchaeota/Crenarchaeota split. Moreover, although TACK superphylum members (Thaumarchaeota, Aigarchaeota, Crenarchaeota, Korarchaeota) [31] encode polB1/Dpo1, they all apart from Crenarchaeota also contain the euryarchaeotal-like PolD [16]. Such confusing evolutionary histories within the archaea are likely to result from accelerated rates of evolution in some clades, alongside significant horizontal gene transfer [15].

Although significant research has uncovered the core components of the archaeal DNA replication and repair pathways, and how they relate to and regulate DNA polymerases, many questions remain. Current advances in archaeal genetic manipulation and biochemical techniques allowing reconstitution and study of at least parts of replisomes are making great strides in elucidating replication and repair mechanisms. This review intends to set out the current status of DNA polymerase research in archaeal DNA replication and repair, and suggests potential future directions for the field.

\section{Archaeal DNA polymerases at the replication fork}

DNA replication is initiated at replication origins following recruitment of specific proteins [32] such as Orc1/Cdc6 [33] and MCM [34]. DNA polymerases are subsequently recruited and interact with additional enzymes and structural proteins to form the replicase holoenzyme, the multi-protein complex responsible for the semi-discontinuous synthesis of both leading and lagging strands of DNA. Although well characterised in bacteria and eukaryotes [35], little is known of the proteins that support the archaeal DNA polymerase holoenzyme. New advances in biochemistry, structural biology, and archaeal genetics in both the Eury- and Crenarchaeota are helping to fill in many of the missing gaps in our knowledge $[36,37]$.

\section{Euryarchaeotal replication: current status}

The Euryarchaeota is a diverse archaeal phylum encompassing halophiles, methanogens and hyperthermophiles, with multiple model species being used to study replication [36]. At first 
glance studying multiple systems may seem redundant, but this is useful considering the evolutionary distance and genetic and phenotypic diversity exhibited by this phylum. Although methanogen and halophile systems have been available for some time, tractable genetic systems have only recently become available for Thermococcus kodakarensis [38], of particular importance as much prior biochemical analysis of euryarchaeotal replication focused on related Thermococcus and Pyrococcus species.

Analysis of the euryarchaeotal replication fork suggests a coordinated action of PolD and PolB centred around the homotrimeric PCNA 'sliding clamp' or processivity factor (Figure 2A) [39]. PolB interacts with a wide range of replication fork components in addition to PCNA, including RPA and primase [40]. PolD from both Pyrococcus abyssi and T. kodakarensis associates with the GINS complex [27,40], and TkoPolD also binds to DNA ligase [27]. Genetic knockout analysis reveals TkoPolB is not essential for viability but provides resistance to UV irradiation, but the two TkoPolD subunits are absolutely required in the Euryarchaeota and hence, that PolD is the replicative polymerase [41]. Similarly, PolD but not PolB was essential for the distantly related methanogen, Methanococcus maripalidus [42]. However, both the chromosomally-encoded PolB1 and PolD were essential in Halobacterium sp. NRC-1, whilst the plasmid-encoded PolB2 was dispensable [43].

This conflicting genetic data suggests either that PolD is the sole replicative DNA polymerase (i.e. the DNA polymerase responsible for synthesising the bulk of both leading and lagging strands in the genome), or that PolB in fact could be a replicative/leading strand polymerase, but functionally redundant with PolD. However proofreading-deficient TkoPolB mutants do not exhibit increased mutation rates in vivo, arguing against this [41]. This also suggests an additional role for PolB in DNA repair, or in another as yet undetermined but essential process in the Halobacteriales. Recent pre-steady state kinetic data support a role for PolD as a replicative DNA polymerase, as Thermococcus sp. $9^{\circ} \mathrm{N}$ PolD follows a similar polymerisation scheme to other DNA polymerases, albeit with a very slow polymerisation rate and higher error rate than other typical replicative polymerases [44]. However as the authors of this study point out, some PolD kinetic parameters and fidelity could be dependent on additional replisome components in vivo, and furthermore, it is unclear if the PolD 3'-5' exonuclease preference of $\mathrm{Mn}^{2+}$ over $\mathrm{Mg}^{2+}$ influences PolD fidelity in vivo.

P. abyssi PCNA (PabPCNA) is observed to bind PabPolB, requiring a C-terminal PIP (PCNAinteracting protein) box motif [45], and both $\mathrm{N}$ - and C-terminal PIP boxes for PabPoID [46]. A PCNA interaction was not observed however for T. kodakarensis PolB (TkoPolB) from affinity pulldowns [27], although this may reflect a similar requirement for primed DNA to potentiate such physical interactions as with PabPCNA [47]. Both PabPolD and PabPolB can extend DNA primers, with PabPoID requiring PabPCNA for efficient DNA synthesis. PabPolD conversely exhibits DNA strand displacement activity in the absence of PabPCNA, but PabPolB requires PabPCNA for this activity $[47,48]$, with strand displacement activity inhibited for both polymerases in $P$. furiosus by the HPfA1 histone protein [49]. Only PabPolD can extend RNA primers, with RNA strand displacement only occurring for PabPCNA-stimulated PabPolD [47], suggesting a role for PolD in initial extension of primed templates on both leading and lagging strands. Furthermore PabPolB is observed to displace PabPolD from DNA but as PabPolB is inhibited by downstream RNA primers [50], this data suggests displacement is likely to occur only on the leading strand. This suggested PabPolD 
initially extends the leading strand RNA primer, prior to switching to PabPolB/PabPCNA for processive synthesis, with PabPolD/PabPCNA involved in lagging strand Okazaki fragment primer extension and maturation.

Recent multiplex capillary electrophoresis analysis with Thermococcus sp. $9^{\circ} \mathrm{N}$ proteins however indicates a requirement for PolB in Okazaki fragment maturation (Figure 2B), as PolD stops 4 nucleotides before downstream Okazaki fragments, even in the presence of PCNA and other replication factors [51]. PolB then fills in this gap, with its strand displacement activity creating a flap structure subsequently processed by FEN1 and DNA ligase, supporting the observation of PabPolB displacing PabPolD already discussed [50]. This begs the question of how PolB can be dispensible for growth following the knockout studies already described [41,42]. It is feasible that the residual full Okazaki processing observed for Thermococcus sp. $9^{\circ} \mathrm{N}$ in the absence of PolB ( 5\%), could result from the weak strand displacement activity of PolD (also seen for PabPolD [47]), and this could potentially be sufficient to sustain this in PolB deletion mutants in vivo [51].

\section{Crenarchaeotal replication: current status}

Although an evolutionarily broad phylum, the Crenarchaeota are typically thermoacidophiles, with perhaps the most well characterised being the Sulfolobus genus [52]. Sulfolobus solfataricus is the most biochemically characterised crenarchaeote in terms of replication, but S. acidocaldarius $[53,54]$ and S. islandicus [55] have offered more tractable genetic systems. Sulfolobus present a particularly excellent model system for studying DNA archaeal replication, as apart from extremely powerful genetics, their synchronisable cell cycle is the most understood in archaea [56] and their proteins are generally readily expressed in bacterial systems [57]. Furthermore, significant advances have been made in imaging sub-cellular localisation of replication components and nascent DNA using the incorporation of fluorescent nucleoside analogues [58].

The Family B polymerase member Dpo1 has been the major focus for crenarchaeotal DNA polymerases since its first isolation from $S$. acidocaldarius [59]. It was proposed as the crenarchaeotal replicase following its association with other core replication factors such as the heterotrimeric SsoPCNA (Figure 2C) [60]. Furthermore, SsoRFC physically interacts with SsoDpo1 and stimulates both DNA polymerase and $3^{\prime}-5^{\prime}$ exonuclease activities [61] by facilitating SsoDpo1 recruitment to DNA. Orc1/Cdc6 origin initiators also interact with SsoDpo1, stimulating SsoDpo1 DNA binding but inhibiting polymerase activity, with SsoCdc6$1 / 2$ inhibiting 3'-5' exonuclease activity [62]. Moreover, only Dpo1 is required for replication by the rudivirus SIRV2 following knockout studies during S. islandicus infection [63], suggesting viral subversion of the Dpo1 cellular replicase.

The lagging strand maturation ability of S. solfataricus (Figure 2D) was demonstrated from the observations that PCNA1 binds FEN1 endonuclease, PCNA2 binds Dpo1 and PCNA3 binds Lig1 ligase, although PCNA3 also weakly binds FEN1 and Dpo1 [60]. An in vitro Okazaki fragment maturation system was subsequently reconstituted, comprising the PCNA1-2-3 heterotrimer, Dpo1, FEN1 and Lig1 alone [64]. This study demonstrated PCNA-stimulated lagging strand DNA synthesis and RNA primer strand displacement by SsoDpo1, creating a flap structure which could then be cleaved by FEN1, followed by covalent ligation by Lig1 of the upstream and downstream Okazaki fragments [64]. Use of a PCNA1-2-3 fused protein 
confirmed the 'molecular toolbelt' model, where Dpo1, FEN1 and Lig1 are simultaneously coordinated around a single PCNA heterotrimer molecule, with different PCNA subunits driving assembly of multiprotein complexes [64], in contrast to the homotrimeric PCNAs of the Euryarchaeota, which more closely resemble eukaryotic PCNA.

Electron microscopy studies confirm this simultaneous engagement of PCNA by the three separate proteins on separate subunits, all positioned on the front face of PCNA [65], supporting previous $P$. furiosus PCNA-polymerase EM studies [66]. The polymerase contacts PCNA extensively in two regions [65-67], with the PfuPolB C-terminal PIP box directing PCNA interaction [67]. As with the euryarchaeotal PolB polymerases, a C-terminal PIP box was found in SsoDpo1 that was essential for the interaction with SsoPCNA2 [68]. However, this is in addition to the originally identified $\mathrm{N}$-terminal PIP box shown to be important for PCNA2/3 binding [60], suggesting a situation instead similar to the euryarchaeotal PolD family, requiring both $\mathrm{N}$ - and C-terminal PIP boxes for PCNA binding [46]. Small-angle X-ray scattering studies support binding of the SsoDpo1 C-terminal PIP box to PCNA2 in the holoenzyme complex [68], similar to that seen for PfuPolB [67].

Surprisingly, the Dpo1-PCNA1-2-3 holoenzyme supports only distributive rather than processive DNA synthesis, with the SsoDpo1 C-terminal PIP box essential for continual recruitment and exchange of Dpo1 with PCNA2 in the holoenzyme [68]. DNA polymerase exchange is also essential to preventing blockage of the replication fork by DNA lesions in bacterial [69] and eukaryotic systems [70]. Translesion (TLS) polymerases are exchanged with a replicative polymerase from the holoenzyme, allowing lesion bypass followed by switching back to a replicative polymerase. As described, Crenarchaeota encode a Y family TLS polymerase (Dpo4/PolY), with SsoDpo4 and SsoDpo1 physically interacting [71]. In addition to the independent Dpo1-PCNA2 and Dpo4-PCNA1 interactions [72], an additional hydrophobic region of Dpo1 behind the palm subdomain contacts Dpo4, allowing recruitment and stabilisation of an the Dpo1-Dpo4-PCNA1-2-3 holoenzyme and thereby increasing DNA synthesis processivity [73]. Direct contacts between the bacterial Pol III replicative and Pol IV TLS polymerases appear important for polymerase switching [73] with the Dpo1-Dpo4 interface appearing conserved [74], suggesting archaea too exhibit DNA polymerase switching from replicative to translesion synthesis.

DNA polymerase dynamics also appear concentration and temperature dependent, as SsoDpo1 trimerises in the presence of DNA in vitro which increases both polymerase kinetic rate and processivity (Figure 2Cvii) [75]. Such oligomerisation is not unusual in the absence of accessory factors, as some bacterial [76] and eukaryotic DNA polymerases [77] have been noted to have stoichiometries greater than one. SsoDpo1 oligomerisation is suggested to also assist in polymerase/exonuclease switching during proofreading [75]. SsoDpo4 itself dimerises at higher and physiological temperatures [78], however, oligomeric Dpo1 also binds undamaged DNA more strongly than Dpo4 at physiological temperatures, suggesting thermodynamic regulation of replisome assembly. Hence, local polymerase concentration and thermodynamic-mediated oligomerisation could work in tandem with PCNA-mediated replisome assembly for both Dpo1 and Dpo4 switching determining replisome composition over normal or damaged templates [73], however, Dpo1/Dpo4 oligomerisation requires validation in vivo. 
Beyond PCNA acting as a nexus at the replication fork where it coordinates binding of polymerases Dpo1 and Dpo4, proteins involved with Okazaki fragment maturation and some DNA repair enzymes [79], little else is known of the composition of the crenarchaeotal replisome, or if structural components exist analogous to the tau subunit that brings three Pol III units together in the bacterial replisome [35]. Recent studies however using epitopetagged proteins in $S$. acidocaldarius have elucidated two small proteins that interact with Sulfolobus Dpo1, so Dpo1 should be considered as a stable heterotrimer [80]. These Dpo1 (polB1)-binding proteins (PBP1, PBP2) significantly increase the thermostability of SsoDpo1. PBP1 negatively influences Dpo1 strand displacement activity in vitro and encourages Dpo1 release on encountering downstream Okazaki fragments, thereby reducing inefficient DNA re-synthesis. PBP2 enhances DNA synthesis and moderates PBP1 influence on Dpo1 distributive synthesis. Hence crenarchaeotal replicative DNA polymerases may be analogous to the replicative eukaryotic polymerases Pol $\delta$ and Pol $\varepsilon$, requiring small accessory proteins as part of their overall structure [81,82]. However as PBP1 and PBP2 are absent from the Thermoproteales (Figure 1) [80], it is feasible that this is not a universal observation for the Crenarchaeota, or that functionally analogous proteins to PBP1 and PBP2 are yet to be found in these species. Effects of PBP1 or PBP2 on the propensity for Dpo1 oligomerisation are yet to be demonstrated however.

\section{Archaeal DNA polymerases in DNA repair - unanswered questions}

Archaea require DNA polymerases for most repair pathways, likely filling in gaps generated following lesion removal [3]. Replicative DNA polymerases however may encounter lesions prior to their repair, potentially stalling due to their active sites being unable to accommodate bulky lesions [83]. Such discrimination is well studied for archaeal Family B polymerases, with the loop region of the fingers determining accurate genome replicating or error-prone TLS functionality [84]. Replicative polymerases may instead exchange with (often Y-family) TLS polymerases on encountering lesions, as TLS polymerases are better suited for dealing with lesions due to their less spatially-constrained active sites, resulting often in error-free lesion bypass [85].

Family Y DNA polymerases are found in all bacteria and eukaryotes [86], but exhibit a varying phyletic distribution in archaea, presumably following significant lateral gene transfer and deletion events (Figure 1). S. solfataricus Dpo4 [87] is an extremely well-characterised and amenable Y-family model DNA polymerase for biochemical, kinetic and structural studies [88]. SsoDpo4 and its S. acidocaldarius orthologue Dbh [89] are demonstrated to be recruited to lesions in vivo to avoid their incorrect replication [90]. Although SsoDpo4 is exchanged with Dpo1 on PCNA as discussed, little is known of the process of Dpo4 recruitment or if a mechanism similar to recruitment via PCNA mono-ubiquitylation in eukaryotes is possible in archaea [90]. It is also unclear how lesion bypass could occur in those archaea lacking a Family $Y$ polymerases. Recent reports however demonstrate that euryarchaeotal primases are able to bypass different lesions to rescue stalled replication forks [91]. It is unknown if primases are specifically recruited for this purpose, or remain in close association with DNA polymerases throughout the replication cycle [40]. Furthermore, homologous recombination or fork regression mechanisms could also potentially indirectly mediate polymerase lesion bypass [92]. 


\section{Crenarchaeotal Dpo2 and Dpo3 - mysterious repair polymerases?}

Although Dpo1 and Dpo3 are ubiquitous in the Crenarchaeota, phylogenetic analysis suggests Dpo2 presence is widespread, but absent in some Crenarchaeota [15]. Despite initial expectations that Dpo2 and Dpo3 would be inactive from lacking certain otherwise conserved catalytic residues [93], similar to Dpo1 and Dpo4, both Dpo2 and Dpo3 were expressed at the RNA and protein level in S. solfataricus [57]. Moreover, they exhibit DNA polymerase and 3'-5' exonuclease activities [57,94,95], indicating they are bona fide proteins and not pseudogenes. In contrast to SsoDpo1 and SsoDpo4 however, SsoDpo2 and SsoDpo3 bind DNA weakly with limited thermostability [57], although other accounts suggest a higher thermostability for Dpo3 [94]. SsoRFC and SsoPCNA together increased primer extension with SsoDpo1/SsoDpo4, but only weakly for SsoDpo2/SsoDpo3 with no obvious PIP boxes for PCNA interaction observed for the latter two [57]. Further, addition of SsoSSB strongly enhanced primer extension with SsoDpo1 and SsoDpo4 but conversely strongly inhibited SsoDpo2 and SsoDpo3 [57]. This accumulated evidence suggests a major role as replicative DNA polymerases is unlikely for Dpo2 and Dpo3.

As with SsoDpo4, SsoDpo2/Dpo3 however both exhibit significant lesion bypass capabilities and are able to bypass 8-oxoG and hypoxanthine lesions [57]. SsoDpo2 can also bypass uracil lesions and interestingly, SsoDpo3 but not SsoDpo2 can bypass bulky cyclobutane thymine dimer lesions [57]. This is surprising as SsoDpo2 is upregulated by UV damage [96,97], suggesting an unclear function for SsoDpo2 in processing UV-induced lesions. Dpo2 was also observed to be upregulated in $S$. islandicus by the Orc1-2 DNA damage response regulator following exposure to NQO, a DNA adduct forming compound [98]. Furthermore, Dpo2 and Dpo3 may also be involved in BER, as both can synthesise across gaps during in vitro $P$. aerophilum BER [99]. Hence, significant evidence points to an involvement for Dpo2 in DNA repair.

Recent reports suggest a further role for Dpo2 in de novo spacer acquisition during CRISPRmediated immunity. Both Dpo2 and Dpo4 were upregulated in S. islandicus, following spacer acquisition resulting from either infection with the STSV2 monocaudavirus [100], or following overexpression of the Csa3a CRISPR transcriptional regulator [101]. Spacer acquisition is dependent on DNA replication in S. islandicus [102], suggesting an active involvement of Dpo 2 in this process. The homologous recombination-related NurA and HerA genes were also upregulated under the same conditions [100,101], potentially implicating Dpo2 in double-strand break repair (at least in the context of CRISPR), potentially similar to that seen for Pol I in E. coli spacer acquisition [103].

Hence, it appears that Dpo2 and Dpo3 are functionally related, but are unlikely to play a role in replicative DNA synthesis from their non-optimal characteristics. They may instead act as specialised repair polymerases, most likely switching with Dpo1 on encountering specific lesions, potentially being redundant with Dpo4 TLS polymerase. Future genetic analyses on Dpo2/3 should shed further light on their specific roles in genome integrity.

\section{Roles of archaeal DNA polymerases in uracil recognition}

Potentially mutagenic deaminated bases in DNA are a prevalent risk for thermophilic archaea in particular [2]. Both euryarchaeotal [104] and crenarchaeotal [105] replicative 
DNA polymerases are found to stall at uracil and hypoxanthine bases [104], although this is not observed for those from bacteria or eukaryotes [106]. This is most prominent for PolB polymerases, stopping at 4 bases prior to the deaminated base where it is tightly bound in an N-terminal pocket $[14,107]$. Euryarchaeotal PolD polymerases are also inhibited by uracil [108] and more weakly by hypoxanthine [109], potentially by a different mechanism from PolB. Instead, PolD polymerases slow down DNA synthesis rather than stopping at a defined site upstream of the deaminated base, and inhibition may also be mediated by a deaminated base in trans on the non-replicated strand $[108,109]$.

DNA polymerase stalling at these lesions is thought to allow recruitment of BER proteins, preventing the lesion being copied and fixed into the genome as a mutation. Indeed, crosstalk between stalled PolB and PolD polymerases and BER components is observed, although this counter-intuitively inhibits uracil-DNA glycosylase, EndoV and EndoQ $[110,111]$, most likely resulting from the tight binding of uracil preventing access by BER enzymes [111]. This shutdown of BER by sequestering lesions could potentially allow replication fork regression/reversal to form a 'chicken foot' structure [92], thereby restoring the deaminated base across from its correct cognate base. This would allow accurate BER repair potentially using PolB to synthesis across the gap [109]. As described previously however, the situation may be even more complex as euryarchaeotal primases were found to bypass not only oxidative and UV damage-induced lesions, but also can replicate past uracil lesions. This may potentially rescue PolB-stalled replication forks [91], suggesting primase-replicase cross-talk may function to ensure replication proceeds.

\section{Conclusions and remaining questions}

For many years, research on archaeal DNA polymerases focused on their biochemical mechanisms and biotechnological exploitation [5]. Recent advances however in biochemical techniques and genetics have yielded deeper insights into the biology of archaeal DNA polymerases and associated proteins in genome replication and repair [36,37]. In particular, establishment of in vitro systems reconstituting Okazaki fragment on the lagging strand [51,64] and in situ epitope tagging to elucidate additional replisome components [80]. A number of outstanding questions still remain however, in particular, the role of Dpo1 oligomerisation and the regulation of the Dpo4 polymerase switch in Crenarchaeota, and the roles of Dpo2/3 in DNA repair and CRISPR. Although only briefly referred to here, the functional relationship of DNA polymerases with primases certainly deserves greater attention, as although archaeal primases comprise an active area of research $[25,112]$, the switch from primases to polymerases during the replication cycle and the relationship to lesion bypass is still unclear [91].

A further enduring question is whether archaeal DNA polymerases are post-translationally modified to regulate their activities and interactions. Both bacterial [113] and eukaryotic [114] DNA polymerases may be modified, and other archaeal genome integrity factors are observed to undergo regulation by post-translational modification. Notably, in Sulfolobus lysine methylation enhances MCM helicase activity [115], Mre11-Rad50 methylation follows DNA damage [116], and the chromatin protein Alba's DNA binding affinity is regulated by

acetylation [117]. E1/E2/E3 ubiquitin-like modification pathways have been discovered in 
other archaeal species and potentially associated with protein turnover $[118,119]$. Hence, it would be interesting to assess if any such modifications play similar roles in archaea, as shown for ubiquitin-mediated polymerase switching at the eukaryotic replication fork [90].

This review has focused on archaeal DNA polymerases in a cellular context, however, many archaeal mobile DNA elements and viruses encode poorly characterised DNA polymerases. Mobilisation through horizontal gene transfer has been proposed to drive DNA polymerase evolution in diverse archaeal phyla and eukaryotes $[15,120]$. Although the rudivirus SIRV2 subverts the $S$. solfataricus Dpo1 polymerase for viral replication, viruses encoding DNA polymerases are seen across the archaeal spectrum e.g. infecting halophiles [121,122], thermophiles [123] and methanogens [124]. Such polymerases are not necessarily thought to be active as missing key catalytic motifs, but as has been seen previously for Dpo2/Dpo3 [93], activity may still be present [57] and their presence suggests a role in evolutionary fitness or infection. DNA polymerases found in other genetic elements include the AEP (archaeo-eukaryotic primase) family found in archaeal plasmids [125] and the arCOG04926 Family B polymerases contained within CRISPR-associated archaeal Casposons $[15,126]$. Characterisation of such novel non-cellular DNA polymerases will shed light not only on archaeal virus/plasmid replication and DNA polymerase evolution, but could potentially further drive the development of novel biotechnological reagents. The future is certainly bright for archaeal DNA polymerase research.

\title{
Summary
}

- Advances in biochemical analysis, structural biology and model system genetics have been pivotal in understanding the functional roles of archaeal DNA polymerases.

- Knowledge of the archaeal replisome protein complement is improving, but more work is needed to further understand DNA polymerase recruitment and regulation.

- Roles of euryarchaeotal PolB/PolD are becoming understood, but their relative function in leading/lagging strand synthesis is still not completely clear.

- Full in vivo roles of crenarchaeotal DNA polymerase oligomerisation are unknown.

- Functions of Dpo2 and Dpo3 in crenarchaeota need to be fully established.

\begin{abstract}
Abbreviations
BER, base excision repair; CRISPR, clustered regularly Interspaced short palindromic repeats; EM, electron microscopy; Pab, Pyrococcus abyssi; Pfu, Pyrococcus furiosus; PIP, PCNA interacting protein; PCR polymerase chain reaction; RFC, replication factor C; Sso, Sulfolobus solfataricus; Tko, Thermococcus kodakarensis; TLS, translesion synthesis;
\end{abstract}

\section{Acknowledgements}

The author is grateful for funding from the University of Huddersfield.

\section{Competing interests}

The author declares no competing interests associated with this study. 


\section{Figure legends}

\section{Figure 1. Phyletic distribution of archaeal DNA polymerases}

Distribution of DNA polymerases and associated structural/regulatory subunits in representative archaeal genomes. Filled circles represent gene presence with colour reflecting the respective text for species. Letters in the first column reflect phylum ( $\mathrm{K}$, Korarchaeota; C, Crenarchaeota; A, Aigarchaeota; T, Thaumarchaeota; N, Nanoarchaeota; E, Euryarchaeota). Species text colouring reflects taxonomic order, apart from for the Thaumarchaeota. Numbers beside filled circles represent number of paralogue copies found in genomes. Filled circles positioned between two columns represent fused genes. Phyletic data retrieved from Raymann et al. [16], Makarova et al. [15], Yan et al. (PBP1/2) [80] and Liu et al. (PriX) [23]. Dpo4/polY distributions were determined by the author using BLASTP homology searches [127], querying S. solfataricus P2 Dpo4 (AAK42588.1) against the nonredundant $\mathrm{NCBI}$ database. BLAST hits were compared against the CDD database [128] to confirm identity as Family Y polymerases.

\section{Figure 2. Current models of archaeal replication fork enzymology}

Key denotes proteins, $\mathrm{X}$ denotes DNA lesions and transparency/dotted outlines represent unconfirmed, tentative or speculative observations. Double-headed dotted arrows represent potentially reversible/dynamic binding interactions. Proteins are not to scale. A. Euryarchaeota leading strand replication. (i) RFC and homotrimeric PCNA are recruited to primed template DNA at replication fork, similar to $2 \mathrm{Ci}$. (ii) PolD binds PCNA. (iii) PolD synthesises DNA from RNA primed template. PolB may potentially be used for continued DNA synthesis from DNA primer, but is redundant in some species. (iv) On encountering DNA lesions (X), DNA primase (PriL/PriS) may bypass the lesion, before DNA polymerase continues elongation. B. Euryarchaeota lagging strand replication. (i) PolD binds to PCNA and elongates from an RNA primer in an Okazaki fragment, but stops 4 nucleotides before the downstream Okazaki fragment. (ii) PolB displaces PolD, synthesising the remaining DNA and displacing the RNA primer. (iii) FEN1 cleaves the displaced RNA/DNA 'flap' and PolB synthesises across the gap. (iv) DNA ligase covalently seals the nick. C. Crenarchaeota leading strand replication. (i) and (ii) Heterotrimeric PCNA is loaded onto primed DNA by RFC, requiring ATP hydrolysis. (iii) Dpo1 (heterotrimeric, with PBP1/PBP2 subunits) is recruited to PCNA (PCNA2). (iv) Dpo1 distributively synthesises DNA but RFC can stimulate Dpo1, although it is not clear if RFC rebinds. If the fork encounters lesions (X), Dpo4 is recruited to PCNA1 and can act potentially as a dimer. (v) Dpo4 may bypass the lesion, but the roles of additional translesion Dpo2 and Dpo3 are not fully established. (vi) Dpo1 continues to synthesise once lesion is bypassed, but it is not clear if Dpo4 leaves the holoenzyme or has a more stable association. (vii) Dpo1 may trimerise, increasing activity and processivity (red arrow), although it is not yet established in vivo or if PBP1/PBP2 or PCNA are present on Dpo1 trimerisation. D. Crenarchaeota lagging strand replication. (i) Heterotrimeric Dpo1 binds PCNA2, FEN1 endonuclease to PCNA1 and LIG1 DNA ligase to PCNA3 in a 'molecular toolbelt'. (ii) Dpo1 synthesises from an RNA primer and displaces the downstream RNA primer, creating an RNA/DNA 'flap'. (iii) FEN1 cleaves the 'flap' and once Dpo1 has synthesised to the downstream DNA, LIG1 covalently seals the nick. 

Calif.

2 Lindahl, T. and Nyberg, B. (1974) Heat-induced deamination of cytosine residues in deoxyribonucleic acid. Biochemistry. 13, 3405-3410

3 White, M.F. and Allers, T. (2018) DNA repair in the archaea-an emerging picture. FEMS Microbiol Rev. 42, 514-526

4 Bessman, M.J., Kornberg, A., Lehman, I.R. and Simms, E.S. (1956) Enzymic synthesis of deoxyribonucleic acid. Biochim Biophys Acta. 21, 197-198

5 Zhang, L., Kang, M., Xu, J. and Huang, Y. (2015) Archaeal DNA polymerases in biotechnology. Appl Microbiol Biotechnol. 99, 6585-6597

6 Wang, Y., Prosen, D.E., Mei, L., Sullivan, J.C., Finney, M. and Vander Horn, P.B. (2004) A novel strategy to engineer DNA polymerases for enhanced processivity and improved performance in vitro. Nucleic Acids Res. 32, 1197-1207

7 Wynne, S.A., Pinheiro, V.B., Holliger, P. and Leslie, A.G. (2013) Structures of an apo and a binary complex of an evolved archeal B family DNA polymerase capable of synthesising highly cy-dye labelled DNA. PLoS One. 8, e70892

8 Chim, N., Shi, C., Sau, S.P., Nikoomanzar, A. and Chaput, J.C. (2017) Structural basis for TNA synthesis by an engineered TNA polymerase. Nat Commun. 8, 1810

9 Chen, C.Y. (2014) DNA polymerases drive DNA sequencing-by-synthesis technologies: both past and present. Front Microbiol. 5, 305

10 Wang, T.S., Wong, S.W. and Korn, D. (1989) Human DNA polymerase alpha: predicted functional domains and relationships with viral DNA polymerases. FASEB J. 3, 14-21

11 Blanco, L., Bernad, A., Blasco, M.A. and Salas, M. (1991) A general structure for DNAdependent DNA polymerases. Gene. 100, 27-38.

12 Guo, J., Zhang, W., Coker, A.R., Wood, S.P., Cooper, J.B., Ahmad, S., et al. (2017) Structure of the family B DNA polymerase from the hyperthermophilic archaeon Pyrobaculum calidifontis. Acta Crystallogr D Struct Biol. 73, 420-427

13 Kropp, H.M., Betz, K., Wirth, J., Diederichs, K. and Marx, A. (2017) Crystal structures of ternary complexes of archaeal B-family DNA polymerases. PLoS One. 12, e0188005

14 Firbank, S.J., Wardle, J., Heslop, P., Lewis, R.J. and Connolly, B.A. (2008) Uracil recognition in archaeal DNA polymerases captured by X-ray crystallography. J Mol Biol. 381, 529-539

15 Makarova, K.S., Krupovic, M. and Koonin, E.V. (2014) Evolution of replicative DNA polymerases in archaea and their contributions to the eukaryotic replication machinery. Front Microbiol. 5, 354

16 Raymann, K., Forterre, P., Brochier-Armanet, C. and Gribaldo, S. (2014) Global phylogenomic analysis disentangles the complex evolutionary history of DNA replication in archaea. Genome Biol Evol. 6, 192-212

17 Kulaeva, O.I., Koonin, E.V., McDonald, J.P., Randall, S.K., Rabinovich, N., Connaughton, J.F., et al. (1996) Identification of a DinB/UmuC homolog in the archeon Sulfolobus solfataricus. Mutat Res. 357, 245-253

18 Ishino, Y., Komori, K., Cann, I.K. and Koga, Y. (1998) A novel DNA polymerase family found in Archaea. J Bacteriol. 180, 2232-2236.

19 Killelea, T., Ralec, C., Bosse, A. and Henneke, G. (2014) PCR performance of a thermostable heterodimeric archaeal DNA polymerase. Front Microbiol. 5, 195 
20 Sauguet, L., Raia, P., Henneke, G. and Delarue, M. (2016) Shared active site architecture between archaeal PoID and multi-subunit RNA polymerases revealed by X-ray crystallography. Nat Commun. 7, 12227

21 Desogus, G., Onesti, S., Brick, P., Rossi, M. and Pisani, F.M. (1999) Identification and characterization of a DNA primase from the hyperthermophilic archaeon Methanococcus jannaschii. Nucleic Acids Res. 27, 4444-4450

22 Lao-Sirieix, S.H. and Bell, S.D. (2004) The heterodimeric primase of the hyperthermophilic archaeon Sulfolobus solfataricus possesses DNA and RNA primase, polymerase and 3'-terminal nucleotidyl transferase activities. J Mol Biol. 344, 1251-1263

23 Liu, B., Ouyang, S., Makarova, K.S., Xia, Q., Zhu, Y., Li, Z., et al. (2015) A primase subunit essential for efficient primer synthesis by an archaeal eukaryotic-type primase. Nat Commun. 6, 7300

24 Chemnitz Galal, W., Pan, M., Kelman, Z. and Hurwitz, J. (2012) Characterization of DNA primase complex isolated from the archaeon, Thermococcus kodakaraensis. J Biol Chem. 287, 16209-16219

25 Yan, J., Holzer, S., Pellegrini, L. and Bell, S.D. (2018) An archaeal primase functions as a nanoscale caliper to define primer length. Proc Natl Acad Sci U S A. 115, 6697-6702

26 Zuo, Z., Rodgers, C.J., Mikheikin, A.L. and Trakselis, M.A. (2010) Characterization of a functional DnaG-type primase in archaea: implications for a dual-primase system. J Mol Biol. 397, 664-676

27 Li, Z., Santangelo, T.J., Cubonova, L., Reeve, J.N. and Kelman, Z. (2010) Affinity purification of an archaeal DNA replication protein network. MBio. 1

28 Walter, P., Klein, F., Lorentzen, E., Ilchmann, A., Klug, G. and Evguenieva-Hackenberg, E. (2006) Characterization of native and reconstituted exosome complexes from the hyperthermophilic archaeon Sulfolobus solfataricus. Mol Microbiol. 62, 1076-1089

29 She, Q., Singh, R.K., Confalonieri, F., Zivanovic, Y., Allard, G., Awayez, M.J., et al. (2001) The complete genome of the crenarchaeon Sulfolobus solfataricus P2. Proc Natl Acad Sci U S A. 98, 7835-7840

30 Daimon, K., Ishino, S., Imai, N., Nagumo, S., Yamagami, T., Matsukawa, H., et al. (2018) Two Family B DNA Polymerases From Aeropyrum pernix, Based on Revised Translational Frames. Front Mol Biosci. 5, 37

31 Martijn, J. and Ettema, T.J. (2013) From archaeon to eukaryote: the evolutionary dark ages of the eukaryotic cell. Biochem Soc Trans. 41, 451-457

32 Robinson, N.P. and Bell, S.D. (2005) Origins of DNA replication in the three domains of life. FEBS J. 272, 3757-3766

33 Dueber, E.C., Costa, A., Corn, J.E., Bell, S.D. and Berger, J.M. (2011) Molecular determinants of origin discrimination by Orc1 initiators in archaea. Nucleic Acids Res. 39, 3621-3631

34 Samson, R.Y., Abeyrathne, P.D. and Bell, S.D. (2016) Mechanism of Archaeal MCM Helicase Recruitment to DNA Replication Origins. Mol Cell. 61, 287-296

35 Yao, N. and O'Donnell, M. (2016) Bacterial and Eukaryotic Replisome Machines. JSM Biochem Mol Biol. 3

36 Farkas, J.A., Picking, J.W. and Santangelo, T.J. (2013) Genetic techniques for the archaea. Annu Rev Genet. 47, 539-561

37 Zatopek, K.M., Gardner, A.F. and Kelman, Z. (2018) Archaeal DNA replication and repair: new genetic, biophysical and molecular tools for discovering and characterizing enzymes, pathways and mechanisms. FEMS Microbiol Rev. 42, 477-488 
38 Hileman, T.H. and Santangelo, T.J. (2012) Genetics Techniques for Thermococcus kodakarensis. Front Microbiol. 3, 195

39 Pan, M., Santangelo, T.J., Cubonova, L., Li, Z., Metangmo, H., Ladner, J., et al. (2013) Thermococcus kodakarensis has two functional PCNA homologs but only one is required for viability. Extremophiles. 17, 453-461

40 Pluchon, P.F., Fouqueau, T., Creze, C., Laurent, S., Briffotaux, J., Hogrel, G., et al. (2013) An extended network of genomic maintenance in the archaeon Pyrococcus abyssi highlights unexpected associations between eucaryotic homologs. PLoS One. 8, e79707

41 Cubonova, L., Richardson, T., Burkhart, B.W., Kelman, Z., Connolly, B.A., Reeve, J.N., et al. (2013) Archaeal DNA polymerase D but not DNA polymerase $B$ is required for genome replication in Thermococcus kodakarensis. J Bacteriol. 195, 2322-2328

42 Sarmiento, F., Mrazek, J. and Whitman, W.B. (2013) Genome-scale analysis of gene function in the hydrogenotrophic methanogenic archaeon Methanococcus maripaludis. Proc Natl Acad Sci U S A. 110, 4726-4731

43 Berquist, B.R., DasSarma, P. and DasSarma, S. (2007) Essential and non-essential DNA replication genes in the model halophilic Archaeon, Halobacterium sp. NRC-1. BMC Genet. 8, 31

44 Schermerhorn, K.M. and Gardner, A.F. (2015) Pre-steady-state Kinetic Analysis of a Family D DNA Polymerase from Thermococcus sp. 9 degrees N Reveals Mechanisms for Archaeal Genomic Replication and Maintenance. J Biol Chem. 290, 21800-21810

45 Tori, K., Kimizu, M., Ishino, S. and Ishino, Y. (2007) DNA polymerases BI and D from the hyperthermophilic archaeon Pyrococcus furiosus both bind to proliferating cell nuclear antigen with their C-terminal PIP-box motifs. J Bacteriol. 189, 5652-5657

46 Castrec, B., Rouillon, C., Henneke, G., Flament, D., Querellou, J. and Raffin, J.P. (2009) Binding to PCNA in Euryarchaeal DNA Replication requires two PIP motifs for DNA polymerase D and one PIP motif for DNA polymerase B. J Mol Biol. 394, 209-218

47 Henneke, G., Flament, D., Hubscher, U., Querellou, J. and Raffin, J.P. (2005) The hyperthermophilic euryarchaeota Pyrococcus abyssi likely requires the two DNA polymerases $D$ and $B$ for DNA replication. J Mol Biol. 350, 53-64

48 Castillo-Lizardo, M., Henneke, G. and Viguera, E. (2014) Replication slippage of the thermophilic DNA polymerases B and D from the Euryarchaeota Pyrococcus abyssi. Front Microbiol. 5, 403

49 Sun, F. and Huang, L. (2016) A euryarchaeal histone modulates strand displacement synthesis by replicative DNA polymerases. Sci China Life Sci. 59, 709-716

50 Rouillon, C., Henneke, G., Flament, D., Querellou, J. and Raffin, J.P. (2007) DNA polymerase switching on homotrimeric PCNA at the replication fork of the euryarchaea Pyrococcus abyssi. J Mol Biol. 369, 343-355

51 Greenough, L., Kelman, Z. and Gardner, A.F. (2015) The roles of family B and D DNA polymerases in Thermococcus species 9 degrees $\mathrm{N}$ Okazaki fragment maturation. J Biol Chem. 290, 12514-12522

52 Quehenberger, J., Shen, L., Albers, S.V., Siebers, B. and Spadiut, O. (2017) Sulfolobus A Potential Key Organism in Future Biotechnology. Front Microbiol. 8, 2474

53 Wagner, M., van Wolferen, M., Wagner, A., Lassak, K., Meyer, B.H., Reimann, J., et al. (2012) Versatile Genetic Tool Box for the Crenarchaeote Sulfolobus acidocaldarius. Front Microbiol. 3, 214 
54 Suzuki, S. and Kurosawa, N. (2017) Development of the Multiple Gene Knockout System with One-Step PCR in Thermoacidophilic Crenarchaeon Sulfolobus acidocaldarius. Archaea. 2017, 7459310

55 Zhang, C., Tian, B., Li, S., Ao, X., Dalgaard, K., Gokce, S., et al. (2013) Genetic manipulation in Sulfolobus islandicus and functional analysis of DNA repair genes. Biochem Soc Trans. 41, 405-410

56 Lindas, A.C. and Bernander, R. (2013) The cell cycle of archaea. Nat Rev Microbiol. 11, 627-638

57 Choi, J.Y., Eoff, R.L., Pence, M.G., Wang, J., Martin, M.V., Kim, E.J., et al. (2011) Roles of the four DNA polymerases of the crenarchaeon Sulfolobus solfataricus and accessory proteins in DNA replication. J Biol Chem. 286, 31180-31193

58 Gristwood, T., Duggin, I.G., Wagner, M., Albers, S.V. and Bell, S.D. (2012) The subcellular localization of Sulfolobus DNA replication. Nucleic Acids Res. 40, 5487-5496

59 Klimczak, L.J., Grummt, F. and Burger, K.J. (1985) Purification and characterization of DNA polymerase from the archaebacterium Sulfolobus acidocaldarius. Nucleic Acids Res. 13, 5269-5282

60 Dionne, I., Nookala, R.K., Jackson, S.P., Doherty, A.J. and Bell, S.D. (2003) A heterotrimeric PCNA in the hyperthermophilic archaeon Sulfolobus solfataricus. Mol Cell. 11, 275-282

61 Xing, X., Zhang, L., Guo, L., She, Q. and Huang, L. (2014) Sulfolobus replication factor C stimulates the activity of DNA polymerase B1. J Bacteriol. 196, 2367-2375

62 Zhang, L., Zhang, L., Liu, Y., Yang, S., Gao, C., Gong, H., et al. (2009) Archaeal eukaryote-like Orc1/Cdc6 initiators physically interact with DNA polymerase B1 and regulate its functions. Proc Natl Acad Sci U S A. 106, 7792-7797

63 Martinez-Alvarez, L., Deng, L. and Peng, X. (2017) Formation of a Viral Replication Focus in Sulfolobus Cells Infected by the Rudivirus Sulfolobus islandicus Rod-Shaped Virus 2. J Virol. 91

64 Beattie, T.R. and Bell, S.D. (2012) Coordination of multiple enzyme activities by a single PCNA in archaeal Okazaki fragment maturation. EMBO J. 31, 1556-1567

65 Cannone, G., Xu, Y., Beattie, T.R., Bell, S.D. and Spagnolo, L. (2015) The architecture of an Okazaki fragment-processing holoenzyme from the archaeon Sulfolobus solfataricus. Biochem J. 465, 239-245

66 Mayanagi, K., Kiyonari, S., Nishida, H., Saito, M., Kohda, D., Ishino, Y., et al. (2011) Architecture of the DNA polymerase B-proliferating cell nuclear antigen (PCNA)-DNA ternary complex. Proc Natl Acad Sci U S A. 108, 1845-1849

67 Nishida, H., Mayanagi, K., Kiyonari, S., Sato, Y., Oyama, T., Ishino, Y., et al. (2009) Structural determinant for switching between the polymerase and exonuclease modes in the PCNA-replicative DNA polymerase complex. Proc Natl Acad Sci U S A. 106, 20693-20698

68 Bauer, R.J., Wolff, I.D., Zuo, X., Lin, H.K. and Trakselis, M.A. (2013) Assembly and distributive action of an archaeal DNA polymerase holoenzyme. J Mol Biol. 425, 4820-4836 69 Kath, J.E., Jergic, S., Heltzel, J.M., Jacob, D.T., Dixon, N.E., Sutton, M.D., et al. (2014) Polymerase exchange on single DNA molecules reveals processivity clamp control of translesion synthesis. Proc Natl Acad Sci U S A. 111, 7647-7652

70 Liu, F., Yang, Y. and Zhou, Y. (2018) Polymerase Delta in Eukaryotes: How is It Transiently Exchanged with Specialized DNA Polymerases During Translesion DNA Synthesis? Curr Protein Pept Sci. 19, 790-804 
71 De Felice, M., Medagli, B., Esposito, L., De Falco, M., Pucci, B., Rossi, M., et al. (2007) Biochemical evidence of a physical interaction between Sulfolobus solfataricus B-family and Y-family DNA polymerases. Extremophiles. 11, 277-282

72 Xing, G., Kirouac, K., Shin, Y.J., Bell, S.D. and Ling, H. (2009) Structural insight into recruitment of translesion DNA polymerase Dpo4 to sliding clamp PCNA. Mol Microbiol. 71, 678-691

73 Cranford, M.T., Chu, A.M., Baguley, J.K., Bauer, R.J. and Trakselis, M.A. (2017) Characterization of a coupled DNA replication and translesion synthesis polymerase supraholoenzyme from archaea. Nucleic Acids Res. 45, 8329-8340

74 Scotland, M.K., Heltzel, J.M., Kath, J.E., Choi, J.S., Berdis, A.J., Loparo, J.J., et al. (2015) A Genetic Selection for dinB Mutants Reveals an Interaction between DNA Polymerase IV and the Replicative Polymerase That Is Required for Translesion Synthesis. PLoS Genet. 11, e1005507

75 Mikheikin, A.L., Lin, H.K., Mehta, P., Jen-Jacobson, L. and Trakselis, M.A. (2009) A trimeric DNA polymerase complex increases the native replication processivity. Nucleic Acids Res. 37, 7194-7205

76 Bailey, M.F., Van der Schans, E.J. and Millar, D.P. (2007) Dimerization of the Klenow fragment of Escherichia coli DNA polymerase $I$ is linked to its mode of DNA binding. Biochemistry. 46, 8085-8099

77 Zahn, K.E., Averill, A.M., Aller, P., Wood, R.D. and Doublie, S. (2015) Human DNA polymerase theta grasps the primer terminus to mediate DNA repair. Nat Struct Mol Biol. 22, 304-311

78 Lin, H.K., Chase, S.F., Laue, T.M., Jen-Jacobson, L. and Trakselis, M.A. (2012) Differential temperature-dependent multimeric assemblies of replication and repair polymerases on DNA increase processivity. Biochemistry. 51, 7367-7382

79 Dionne, I. and Bell, S.D. (2005) Characterization of an archaeal family 4 uracil DNA glycosylase and its interaction with PCNA and chromatin proteins. Biochem J. 387, 859-863

80 Yan, J., Beattie, T.R., Rojas, A.L., Schermerhorn, K., Gristwood, T., Trinidad, J.C., et al. (2017) Identification and characterization of a heterotrimeric archaeal DNA polymerase holoenzyme. Nat Commun. 8, 15075

81 Tahirov, T.H. (2012) Structure and function of eukaryotic DNA polymerase delta. Subcell Biochem. 62, 217-236

82 Hogg, M. and Johansson, E. (2012) DNA polymerase epsilon. Subcell Biochem. 62, 237-257

83 Hogg, M., Wallace, S.S. and Doublie, S. (2005) Bumps in the road: how replicative DNA polymerases see DNA damage. Curr Opin Struct Biol. 15, 86-93

84 Jozwiakowski, S.K., Keith, B.J., Gilroy, L., Doherty, A.J. and Connolly, B.A. (2014) An archaeal family-B DNA polymerase variant able to replicate past DNA damage: occurrence of replicative and translesion synthesis polymerases within the B family. Nucleic Acids Res. 42, 9949-9963

85 Broyde, S., Wang, L., Rechkoblit, O., Geacintov, N.E. and Patel, D.J. (2008) Lesion processing: high-fidelity versus lesion-bypass DNA polymerases. Trends Biochem Sci. 33, 209-219

86 Monttinen, H.A., Ravantti, J.J., Stuart, D.I. and Poranen, M.M. (2014) Automated structural comparisons clarify the phylogeny of the right-hand-shaped polymerases. Mol Biol Evol. 31, 2741-2752 
87 Boudsocq, F., Iwai, S., Hanaoka, F. and Woodgate, R. (2001) Sulfolobus solfataricus P2 DNA polymerase IV (Dpo4): an archaeal DinB-like DNA polymerase with lesion-bypass properties akin to eukaryotic poleta. Nucleic Acids Res. 29, 4607-4616

88 Maxwell, B.A. and Suo, Z. (2014) Recent insight into the kinetic mechanisms and conformational dynamics of Y-Family DNA polymerases. Biochemistry. 53, 2804-2814

89 Sakofsky, C.J., Foster, P.L. and Grogan, D.W. (2012) Roles of the Y-family DNA polymerase $\mathrm{Dbh}$ in accurate replication of the Sulfolobus genome at high temperature. DNA Repair (Amst). 11, 391-400

90 Sakofsky, C.J. and Grogan, D.W. (2015) Lesion-Induced Mutation in the Hyperthermophilic Archaeon Sulfolobus acidocaldarius and Its Avoidance by the Y-Family DNA Polymerase Dbh. Genetics. 201, 513-523

91 Jozwiakowski, S.K., Borazjani Gholami, F. and Doherty, A.J. (2015) Archaeal replicative primases can perform translesion DNA synthesis. Proc Natl Acad Sci U S A. 112, E633-638

92 Grogan, D.W. (2015) Understanding DNA Repair in Hyperthermophilic Archaea: Persistent Gaps and Other Reasons to Focus on the Fork. Archaea. 2015, 942605

93 Rogozin, I.B., Makarova, K.S., Pavlov, Y.I. and Koonin, E.V. (2008) A highly conserved family of inactivated archaeal B family DNA polymerases. Biol Direct. 3, 32

94 Bauer, R.J., Begley, M.T. and Trakselis, M.A. (2012) Kinetics and fidelity of polymerization by DNA polymerase III from Sulfolobus solfataricus. Biochemistry. 51, 19962007

95 Ralec, C., Henry, E., Lemor, M., Killelea, T. and Henneke, G. (2017) Calcium-driven DNA synthesis by a high-fidelity DNA polymerase. Nucleic Acids Res. 45, 12425-12440

96 Frols, S., Gordon, P.M., Panlilio, M.A., Duggin, I.G., Bell, S.D., Sensen, C.W., et al. (2007) Response of the hyperthermophilic archaeon Sulfolobus solfataricus to UV damage. J Bacteriol. 189, 8708-8718

97 Gotz, D., Paytubi, S., Munro, S., Lundgren, M., Bernander, R. and White, M.F. (2007) Responses of hyperthermophilic crenarchaea to UV irradiation. Genome Biol. 8, R220

98 Sun, M., Feng, X., Liu, Z., Han, W., Liang, Y.X. and She, Q. (2018) An Orc1/Cdc6 ortholog functions as a key regulator in the DNA damage response in Archaea. Nucleic Acids Res. 46, 6697-6711

99 Sartori, A.A. and Jiricny, J. (2003) Enzymology of base excision repair in the hyperthermophilic archaeon Pyrobaculum aerophilum. J Biol Chem. 278, 24563-24576

100 Leon-Sobrino, C., Kot, W.P. and Garrett, R.A. (2016) Transcriptome changes in STSV2infected Sulfolobus islandicus REY15A undergoing continuous CRISPR spacer acquisition. Mol Microbiol. 99, 719-728

101 Liu, T., Liu, Z., Ye, Q., Pan, S., Wang, X., Li, Y., et al. (2017) Coupling transcriptional activation of CRISPR-Cas system and DNA repair genes by Csa3a in Sulfolobus islandicus. Nucleic Acids Res. 45, 8978-8992

102 Erdmann, S., Le Moine Bauer, S. and Garrett, R.A. (2014) Inter-viral conflicts that exploit host CRISPR immune systems of Sulfolobus. Mol Microbiol. 91, 900-917

103 Ivancic-Bace, I., Cass, S.D., Wearne, S.J. and Bolt, E.L. (2015) Different genome stability proteins underpin primed and naive adaptation in E. coli CRISPR-Cas immunity. Nucleic Acids Res. 43, 10821-10830

104 Greagg, M.A., Fogg, M.J., Panayotou, G., Evans, S.J., Connolly, B.A. and Pearl, L.H. (1999) A read-ahead function in archaeal DNA polymerases detects promutagenic templatestrand uracil. Proc Natl Acad Sci U S A. 96, 9045-9050 
105 Gruz, P., Shimizu, M., Pisani, F.M., De Felice, M., Kanke, Y. and Nohmi, T. (2003) Processing of DNA lesions by archaeal DNA polymerases from Sulfolobus solfataricus. Nucleic Acids Res. 31, 4024-4030

106 Wardle, J., Burgers, P.M., Cann, I.K., Darley, K., Heslop, P., Johansson, E., et al. (2008) Uracil recognition by replicative DNA polymerases is limited to the archaea, not occurring with bacteria and eukarya. Nucleic Acids Res. 36, 705-711

107 Fogg, M.J., Pearl, L.H. and Connolly, B.A. (2002) Structural basis for uracil recognition by archaeal family B DNA polymerases. Nat Struct Biol. 9, 922-927

108 Richardson, T.T., Gilroy, L., Ishino, Y., Connolly, B.A. and Henneke, G. (2013) Novel inhibition of archaeal family-D DNA polymerase by uracil. Nucleic Acids Res. 41, 4207-4218

109 Abellon-Ruiz, J., Waldron, K.J. and Connolly, B.A. (2016) Archaeoglobus Fulgidus DNA Polymerase D: A Zinc-Binding Protein Inhibited by Hypoxanthine and Uracil. J Mol Biol. 428, 2805-2813

110 Emptage, K., O'Neill, R., Solovyova, A. and Connolly, B.A. (2008) Interplay between DNA polymerase and proliferating cell nuclear antigen switches off base excision repair of uracil and hypoxanthine during replication in archaea. J Mol Biol. 383, 762-771

111 Abellon-Ruiz, J., Ishino, S., Ishino, Y. and Connolly, B.A. (2016) Archaeal DNA Polymerase-B as a DNA Template Guardian: Links between Polymerases and Base/Alternative Excision Repair Enzymes in Handling the Deaminated Bases Uracil and Hypoxanthine. Archaea. 2016, 1510938

112 Holzer, S., Yan, J., Kilkenny, M.L., Bell, S.D. and Pellegrini, L. (2017) Primer synthesis by a eukaryotic-like archaeal primase is independent of its Fe-S cluster. Nat Commun. 8, 1718

113 Sutton, M.D., Narumi, I. and Walker, G.C. (2002) Posttranslational modification of the umuD-encoded subunit of Escherichia coli DNA polymerase $V$ regulates its interactions with the beta processivity clamp. Proc Natl Acad Sci U S A. 99, 5307-5312

114 El-Andaloussi, N., Valovka, T., Toueille, M., Steinacher, R., Focke, F., Gehrig, P., et al. (2006) Arginine methylation regulates DNA polymerase beta. Mol Cell. 22, 51-62

115 Xia, Y., Niu, Y., Cui, J., Fu, Y., Chen, X.S., Lou, H., et al. (2015) The Helicase Activity of Hyperthermophilic Archaeal MCM is Enhanced at High Temperatures by Lysine Methylation. Front Microbiol. 6, 1247

116 Kish, A., Gaillard, J.C., Armengaud, J. and Elie, C. (2016) Post-translational methylations of the archaeal Mre11:Rad50 complex throughout the DNA damage response. Mol Microbiol. 100, 362-378

117 Bell, S.D., Botting, C.H., Wardleworth, B.N., Jackson, S.P. and White, M.F. (2002) The interaction of Alba, a conserved archaeal chromatin protein, with Sir2 and its regulation by acetylation. Science. 296, 148-151

118 Hennell James, R., Caceres, E.F., Escasinas, A., Alhasan, H., Howard, J.A., Deery, M.J., et al. (2017) Functional reconstruction of a eukaryotic-like E1/E2/(RING) E3 ubiquitylation cascade from an uncultured archaeon. Nat Commun. 8, 1120

119 Anjum, R.S., Bray, S.M., Blackwood, J.K., Kilkenny, M.L., Coelho, M.A., Foster, B.M., et al. (2015) Involvement of a eukaryotic-like ubiquitin-related modifier in the proteasome pathway of the archaeon Sulfolobus acidocaldarius. Nat Commun. 6, 8163

120 Takemura, M., Yokobori, S. and Ogata, H. (2015) Evolution of Eukaryotic DNA Polymerases via Interaction Between Cells and Large DNA Viruses. J Mol Evol. 81, 24-33 
121 Tang, S.L., Nuttall, S., Ngui, K., Fisher, C., Lopez, P. and Dyall-Smith, M. (2002) HF2: a double-stranded DNA tailed haloarchaeal virus with a mosaic genome. Mol Microbiol. 44, 283-296

122 Bath, C., Cukalac, T., Porter, K. and Dyall-Smith, M.L. (2006) His1 and His2 are distantly related, spindle-shaped haloviruses belonging to the novel virus group, Salterprovirus. Virology. 350, 228-239

123 Wang, H., Guo, Z., Feng, H., Chen, Y., Chen, X., Li, Z., et al. (2017) A novel Sulfolobus virus with an exceptional capsid architecture. J Virol

124 Weidenbach, K., Nickel, L., Neve, H., Alkhnbashi, O.S., Kunzel, S., Kupczok, A., et al. (2017) Methanosarcina Spherical Virus, a Novel Archaeal Lytic Virus Targeting Methanosarcina Strains. J Virol. 91

125 Kazlauskas, D., Sezonov, G., Charpin, N., Venclovas, C., Forterre, P. and Krupovic, M. (2018) Novel Families of Archaeo-Eukaryotic Primases Associated with Mobile Genetic Elements of Bacteria and Archaea. J Mol Biol. 430, 737-750

126 Krupovic, M., Makarova, K.S., Forterre, P., Prangishvili, D. and Koonin, E.V. (2014) Casposons: a new superfamily of self-synthesizing DNA transposons at the origin of prokaryotic CRISPR-Cas immunity. BMC Biol. 12, 36

127 Altschul, S.F., Gish, W., Miller, W., Myers, E.W. and Lipman, D.J. (1990) Basic local alignment search tool. J Mol Biol. 215, 403-410

128 Marchler-Bauer, A., Derbyshire, M.K., Gonzales, N.R., Lu, S., Chitsaz, F., Geer, L.Y., et al. (2015) CDD: NCBI's conserved domain database. Nucleic Acids Res. 43, D222-226 
Unclassified

Sulfolobales

Sulfolobales

Sulfolobales

Sulfolobales

Sulfolobales

Sulfolobales

Desulfurococcales

Desulfurococcales

Desulfurococcales

Desulfurococcales

Desulfurococcales

Desulfurococcale

Desulfurococcales

Desulfurococcales

Acidilobales

Thermoproteales

Thermoproteales

Thermoproteales

Thermoproteales

Thermoproteales

Thermoproteales

Unclassified

Nitrososphaerales

Cenarchaeales

Nitrosopumilales

Nanoarchaeales

DHVE2 group

Thermoplasmatales

Thermoplasmatales

Thermoplasmatales

Thermoplasmatales

Archaeoglobales

Archaeoglobales

Archaeoglobales

Halobacteriales

Halobacteriales

Halobacteriales

Halobacteriales

Halobacteriales

Halobacteriales

Halobacteriales

Halobacteriales

Halobacteriales

Halobacteriales

Halobacteriales

Halobacteriales

Halobacteriales

Halobacteriales

Halobacteriales

Halobacteriales

Halobacteriales

Halobacteriales

Methanomicrobiales Methanocorpusculum labreanum

Methanomicrobiales Methanoculleus bourgensis

Methanomicrobiales Methanoplanuspetrolearius

Methanomicrobiales Methanosphaerula palustris

Methanomicrobiales Methanoregulaboonei

Methanomicrobiales Methanospirillum hungatei

Methanocellales

Methanosarcinales

Methanosarcinales

Methanosarcinales

Methanosarcinales

Methanosarcinales

Methanosarcinales

Methanosarcinales

Methanococcales

Methanococcales

Methanococcales

Methanococcales

Methanococcales

Methanopyrales

Methanobacteriales

Methanobacteriales

Methanobacteriales

Methanobacteriales

Thermococcales

Thermococcales

Thermococcales

Thermococcales

Thermococcales

Thermococcales

Thermococcales
Candidatus Korarchaeum cryptofilum lobus solfataricus

Metallosphaera sedula

Sulfolobus tokodaii

Sulfolobus acidocaldarius

coccus hospitalis

ermus butylicus

Staphylothermus marinus

rmosphaera aggregan

位

aggregans

Acidilobus saccharovorans

ermofilum pendens

Probaculum arsenaticum

Thermoproteus tenax

aldivirga maquilingensis

eta distributa

symbiosum

ciduliprofundum boonei

idophilum

erroplasma acidarmanus

philus torridus

Archaeoglobus profundus

erroglobus placidus

Haloferax volcanii

borinquense

alobacterium salinarum

alalkalicoccus jeotgali

Haloterrigena turkmenica

Natrinema pellirubrum

xanaduensis

Natronobacterium gregoryi

onas pharaonis

Halorhabdus utahensis

Halomicrobium mukohataei

Haloarcula hispanica

Methanospirillum hungate

Methanosaeta harundinacea

Methanosaeta concilii

Methanosaeta thermophila

ethanosarcina barkeri

Methanosarcina acetivorans

Methanosarcina mazei

Methanococcoides burtonii

Methanocaldococcus infernus

Methanocaldococcus jannaschi

ethanothermococcus okinawensis

Methanococcus voltae

Methanococcus maripaludis

Methanopyrus kandleri

Methanothermus fervidus

Methanosphaera stadtmanae

Methanobrevibacter smithii

Methanothermobacter thermautotrophicus

Pyrococcus furiosus

Pyrococcus abyssi

Pyrococcus horikoshii

Thermococcus onnurineus

Thermococcus kodakarensis

Thermococcus barophilus

Thermococcus litoralis

\begin{tabular}{|c|c|c|c|c|c|c|c|c|c|}
\hline $\begin{array}{l}\text { Dpo1 } \\
\text { (polB1) }\end{array}$ & $\begin{array}{c}\text { PBP1 PBP2 } \\
\text { (Dpo1 complex) }\end{array}$ & \begin{tabular}{|c|} 
Dpo2 \\
(polB2)
\end{tabular} & $\begin{array}{l}\text { Dpo3 } \\
\text { (polB3) }\end{array}$ & \begin{tabular}{|l} 
Dpo4 \\
(polY)
\end{tabular} & $\begin{array}{r}\text { DP1 } \\
\text { (pold }\end{array}$ & & $\begin{array}{l}\text { Pris } \\
(p\end{array}$ & $\begin{array}{c}\text { Pril } \\
\text { orimas }\end{array}$ & PriX \\
\hline $\begin{array}{l}0 \\
0 \\
0 \\
0 \\
0 \\
0 \\
0 \\
0 \\
0 \\
0 \\
0 \\
0 \\
0 \\
0 \\
0 \\
0 \\
0 \\
0 \\
0 \\
0 \\
0 \\
0 \\
0 \\
0 \\
0 \\
0 \\
0\end{array}$ & $\begin{array}{ll}\circ & 0 \\
0 & 0 \\
\circ & \circ \\
\circ & 0 \\
\circ & 0 \\
\circ & 0 \\
\circ & 0 \\
\circ & 0 \\
0 & 0 \\
0 & 0 \\
\circ & 0 \\
0 & 0 \\
0 & 0 \\
\circ & 0 \\
0 & 0 \\
0 & 0\end{array}$ & $\begin{array}{l}0 \\
\\
0 \\
0 \\
0 \\
0 \\
0 \\
0 \\
\circ 3 \\
\circ \\
02 \\
0 \\
02 \\
0 \\
0 \\
0 \\
\circ \\
0 \\
0 \\
0 \\
0\end{array}$ & $\begin{array}{l}O \\
0 \\
0 \\
0 \\
0 \\
0 \\
0 \\
0 \\
0 \\
0 \\
0 \\
0 \\
0 \\
0 \\
0 \\
0 \\
0 \\
0 \\
0 \\
0 \\
0 \\
0 \\
0 \\
0 \\
0 \\
0 \\
0 \\
0 \\
\end{array}$ & $\begin{array}{l}0 \\
0 \\
0 \\
0 \\
0 \\
0 \\
0 \\
0 \\
0 \\
0 \\
0 \\
0 \\
0 \\
0 \\
0 \\
0 \\
0 \\
0 \\
0 \\
0 \\
0 \\
0 \\
0 \\
0 \\
0 \\
0 \\
0 \\
0\end{array}$ & 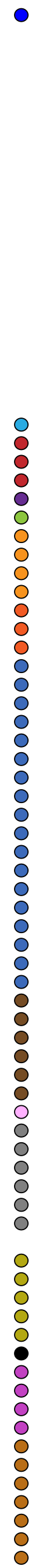 & $\begin{array}{l}0 \\
0 \\
0 \\
0 \\
0 \\
0 \\
0 \\
0 \\
0 \\
0 \\
0 \\
0 \\
0 \\
0 \\
0 \\
0 \\
0 \\
0 \\
0 \\
0 \\
0 \\
0 \\
0 \\
0 \\
0 \\
0 \\
0 \\
0 \\
0 \\
0 \\
0 \\
0 \\
0 \\
0 \\
0 \\
0 \\
0 \\
0 \\
0 \\
0 \\
0 \\
0 \\
0 \\
0 \\
0 \\
0 \\
0 \\
0 \\
0 \\
0 \\
0 \\
0 \\
0 \\
0 \\
0 \\
0 \\
0 \\
0 \\
0 \\
0 \\
0 \\
0 \\
0 \\
0\end{array}$ & $\begin{array}{l}0 \\
0 \\
0 \\
0 \\
0 \\
0 \\
0 \\
0\end{array}$ & $\begin{array}{l}0 \\
0 \\
0 \\
0 \\
0 \\
0 \\
0\end{array}$ & $\begin{array}{l}0 \\
0 \\
0 \\
0 \\
0 \\
0\end{array}$ \\
\hline
\end{tabular}


A

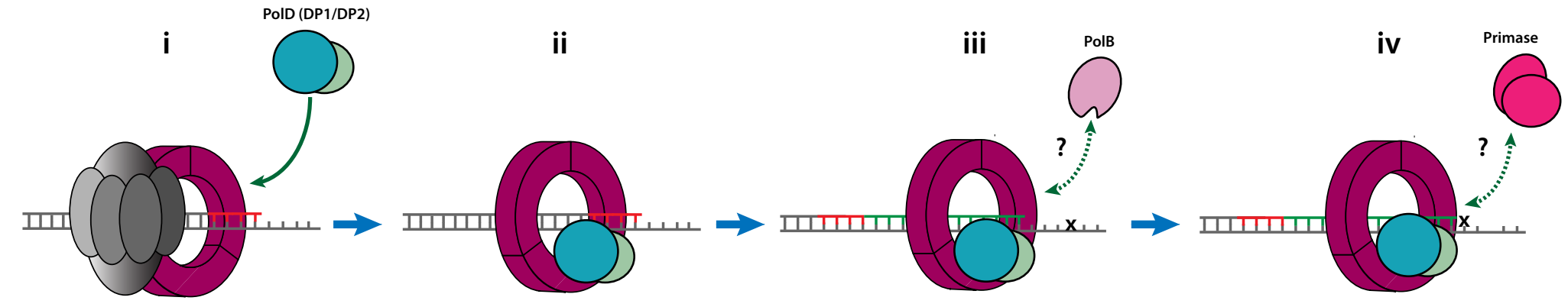

B

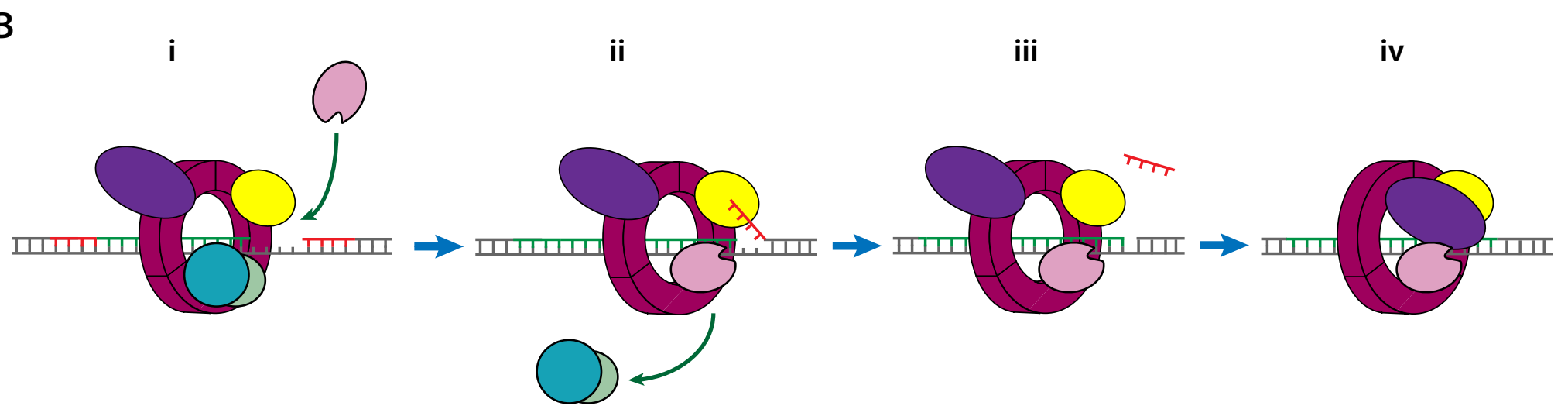

C
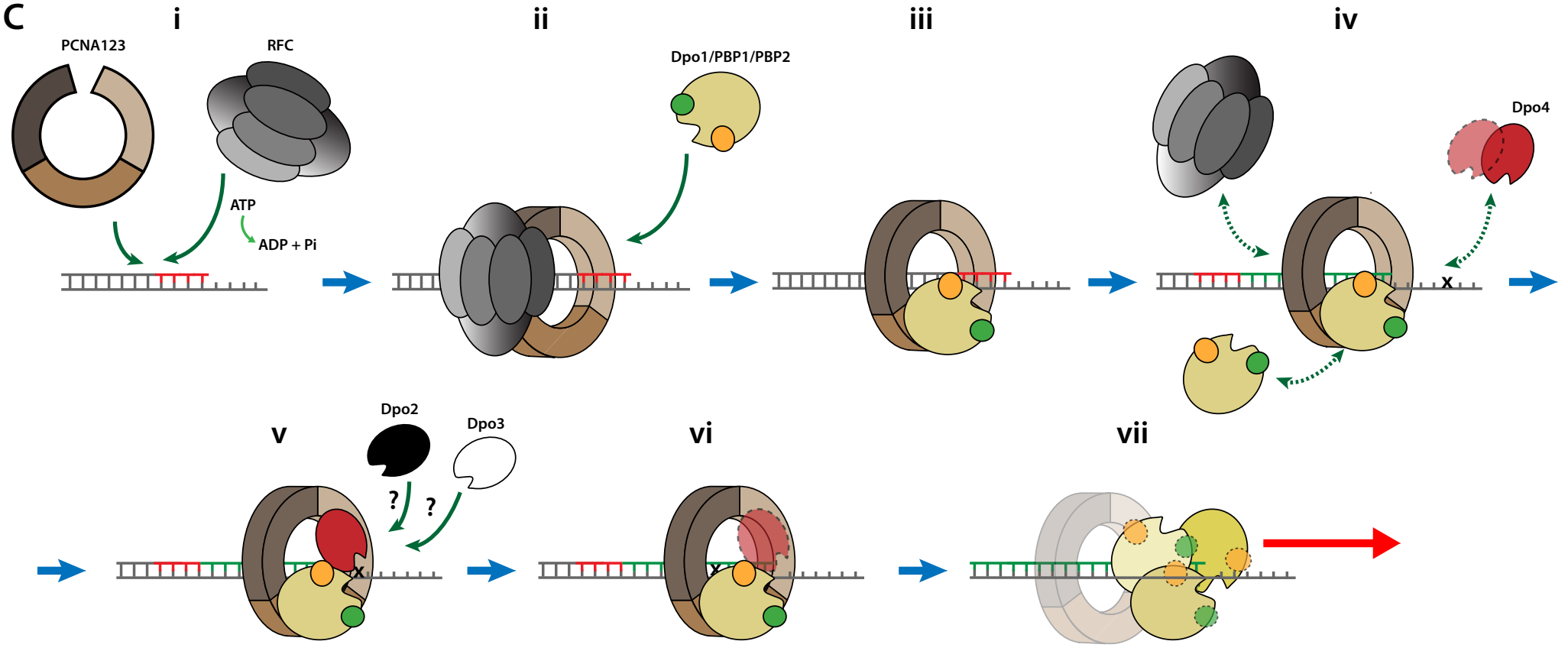

i ii

iii iv
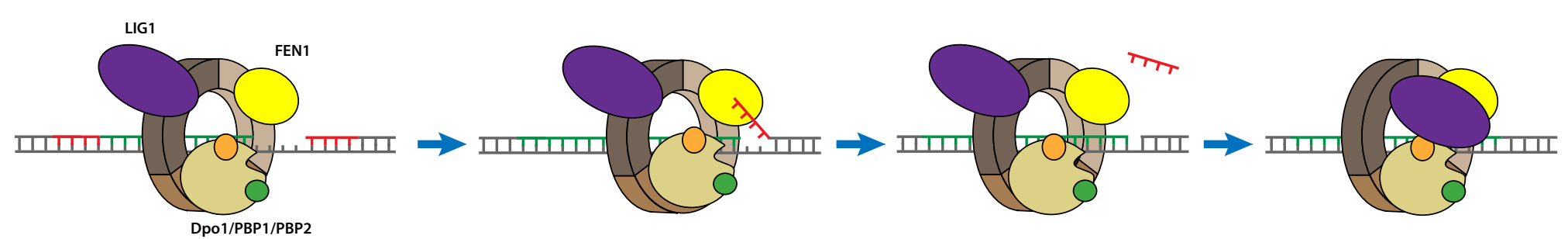

\begin{tabular}{|llll|}
$\square$ PCNA1 & $\square$ Dpo2 polymerase & $\square$ RFC complex & $\square$ DNA ligase \\
$\square$ PCNA2 & $\square$ Dpo3 polymerase & $\square$ PolD DP2 polymerase & $\square$ Primase (PriS/PriL) \\
$\square$ PCNA3 & $\square$ Dpo4 polymerase & $\square$ PolD DP1 exonuclease & T RNA primer \\
$\square$ PCNA (euryarchaeotal) & $\square$ PBP1 (polB1 binding protein 1) & $\square$ PolB & T template DNA \\
$\square$ Dpo1 polymerase & $\square$ PBP2 (polB1 binding protein 2) & $\square$ FEN1 & T nascent DNA \\
\hline
\end{tabular}

